\title{
Electron energy loss spectroscopy of sub-10 nm 2D MoS 2 crystals
}

Pawan Kumar ${ }^{1}$, James Horwath ${ }^{2}$, Surendra Anantharaman ${ }^{1}$, Andrew Meng ${ }^{2}$, Juan Carlos Idrobo ${ }^{3}$, Eric Stach $^{1}$ and Deep Jariwala ${ }^{4}$

${ }^{1}$ University of Pennsylvania, Philadelphia, Pennsylvania, United States, ${ }^{2}$ University of Pennsylvania, United States, ${ }^{3}$ Oak Ridge National Laboratory, Oak Ridge, Tennessee, United States, ${ }^{4}$ University of Pennsylvania, PHILADELPHIA, Pennsylvania, United States

Two-dimensional (2D) transition metal dichalcogenides (TMDCs) have been the subject of intense study due to their outstanding electronic and optical properties.[1] This class of extremely thin materials shows multiple phases and their structural reversal in the extent of an energetic stimulus. These phenomena happen because the atoms can have different orientations within single/multiple layers, or these layers can stack with different rotations.[2]

Here, we measure and analyze the electron energy loss spectra from $2 \mathrm{D}$ crystals with sub-10 nm diameter utilizing a monochromatic electron source. We use an in-situ holder with ultrafast ( $>25 \mathrm{C} / \mathrm{sec}$ ) heating rates to transform the single-crystal sheets into these particles inside the TEM column.[3] We utilized a combination of aberration-corrected scanning transmission electron microscopy (JEOL NEOARM \& Nion Hermes monochromated aberration-corrected (MAC)-STEM) and in-situ heating holder using microfabricated heating elements (Hummingbird Scientific) to directly visualize these transformation processes on the atomic scale. The heating rates and final temperatures which are well-calibrated through various prior techniques allow us to control the size and distribution of these $2 \mathrm{D}$ nanoparticles during the transformation.

Here we investigate two different configurations of TMDC ( $\mathrm{MoS}_{2}$ mono to few layers)) samples prepared via dry stamp transfer techniques. The first configuration consists of a bilayer heterostructure wherein the MoS2 crystal sits on top of a few-layer hexagonal boron nitride that provides superior and uniform heat conduction/transfer. The second configuration is a trilayer heterostructure where the $\mathrm{MoS}_{2}$ single crystal flake is confined (sandwiched) under thin h-BN layers both on the top and bottom. Ultrafast heating (25 ${ }^{\circ} \mathrm{C} / \mathrm{sec}$ ) of these samples leads to the transformation of uniform flat single-crystal $\mathrm{MoS}_{2}$ into highly ordered, crystalline, and mostly hexagonal (mostly) particles of sizes ranging 5-20 nm in diameter as shown in Figure 1. Further, we use, atomic probe (nearly $1 \AA$ ) monochromated source operating at $60 \mathrm{kV}$ to analyze these particles formed in the above two configurations for their valence band energy loss spectroscopy. Figure 1f contains EELS spectra taken for $\mathrm{MoS}_{2}$ crystals in the above two configurations. Spectra are shown for valence band as well as conduction band plasmon range respectively. Clearly, differences between the two cases are observed which will be explained in detail.

The ability to control and create highly confined $\mathrm{MoS}_{2}$ particles leads to exciting new possibilities in materials engineering and electronic structure modulation which could ultimately lead to new device applications of these confined 2D materials and their heterostructures.[4] 

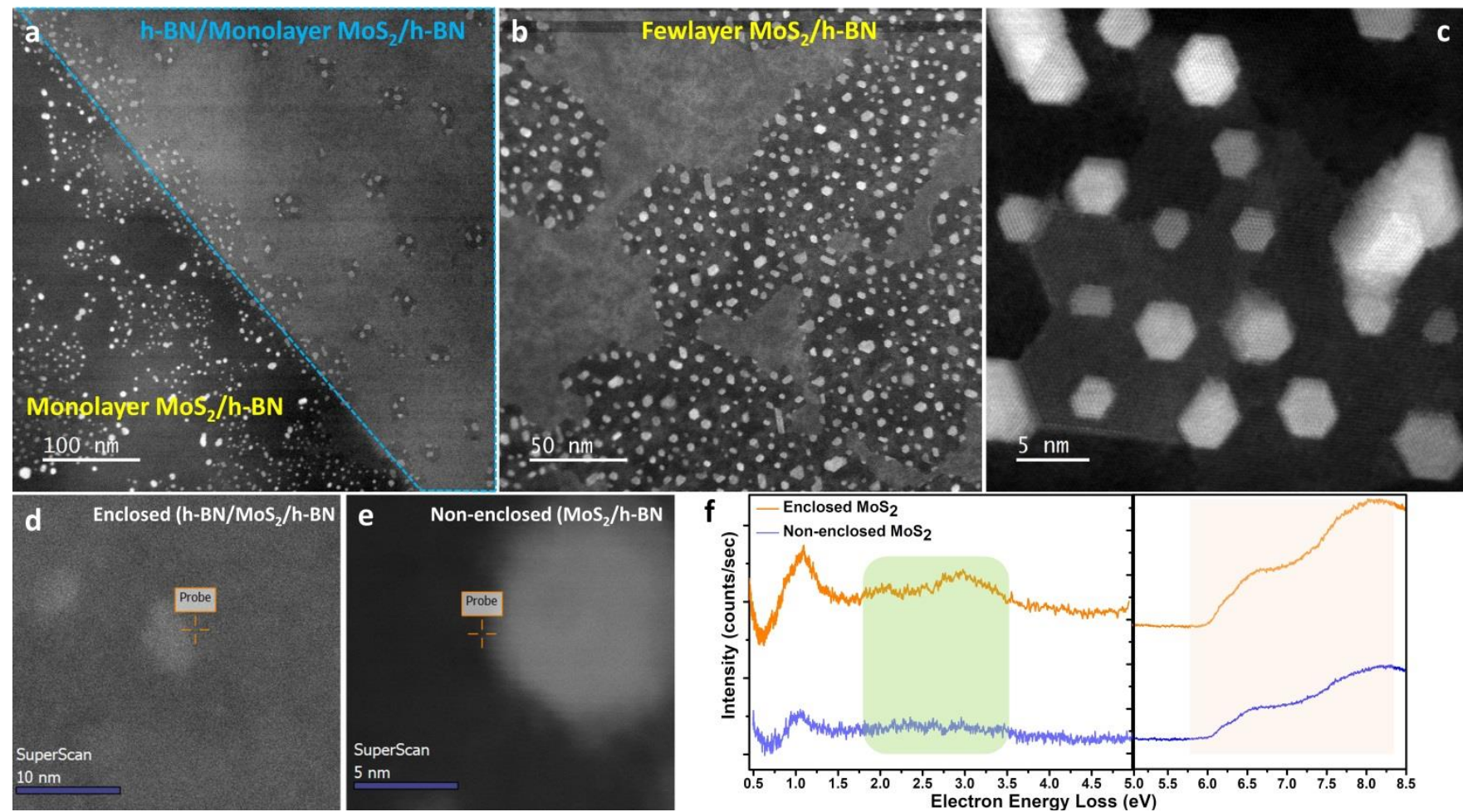

Figure 1. Ultrafast heating at $25^{\circ} \mathrm{C} / \mathrm{sec}$ of MoS2 (a) Low magnification ADF STEM image of the region where marked (light blue) region shows the enclosed (h-BN/MoS2/h-BN) configuration while the rest portion is non-enclosed MoS2. (b) A large aerial view for few-layer MoS2 disintegrated to quantum size particles and corresponding (c) high magnification aberration-corrected STEM image shows the formation of a high degree of crystallinity of disintegrated quantum size MoS2 particles from large 2D sheets, (d, e) $\mathrm{ADF}$ image of the chose quantum MoS2 particle, enclosed as well as non-enclosed configuration for the EELS measurement and corresponding (f) EELS spectrum for two different regions, valence band, and conduction band plasmons.

\section{References}

[1] Novoselov, K. S.; Jiang, D.; Schedin, F.; Booth, T. J.; Khotkevich, V. V.; Morozov, S. V.; Geim, A. K., Two-dimensional atomic crystals. Proceedings of the National Academy of Sciences of the United States of America 2005, 102 (30), 10451-10453.

[2] Lin, Y.-C.; Dumcenco, D. O.; Huang, Y.-S.; Suenaga, K., Atomic mechanism of the semiconductingto-metallic phase transition in single-layered MoS2. Nature Nanotechnology 2014, 9 (5), 391-396.

[3] Kumar, P; Horwath, J. P.; Foucher, A. C.; Price, C. C.; Acero, N.; Shenoy, V. B.; Stach, E. A.; Jariwala, D., Direct visualization of out-of-equilibrium structural transformations in atomically thin chalcogenides 2020, npj 2D Materials and Applications, 4, 1-10.

[4] Price, C. C.; Frey, N. C.; Jariwala, D.; Shenoy, V. B., Engineering Zero-Dimensional Quantum Confinement in Transition-Metal Dichalcogenide Heterostructures. ACS Nano 2019, 13 (7), 8303-8311. 